-Buenos licapida Roja.

-Buenos ciab

A dóndes ar urano, Caperuci

Voy a cas in ata.

QQué llevn tital

- levo a wain pistel y una bot vino. lucestá $n^{4}$ isay coresto cotrara

- Donot in?

- a el e od me fía pyo más jeuarto dor pa Esth deby vo aquell trandes emas, joralonos pote será di ibnocer a qua.

nsoveta de Le er muy thabr

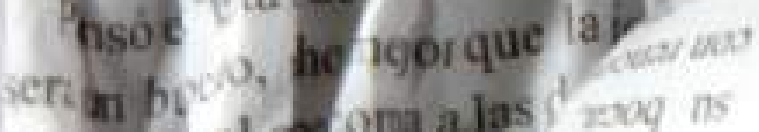
nods, he ial coroma a las xaoq ins odr cor st po cou ast $\operatorname{irsc}_{1} C_{\text {la }} y^{2}$ :

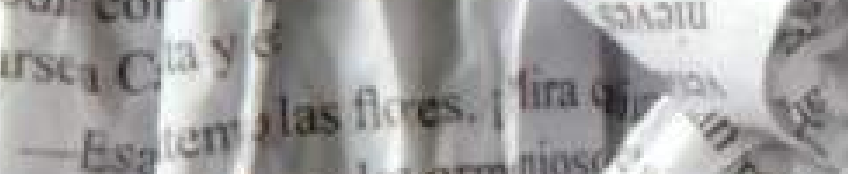

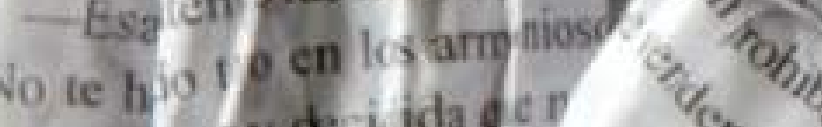
yaras? ay y decilida of 1 .

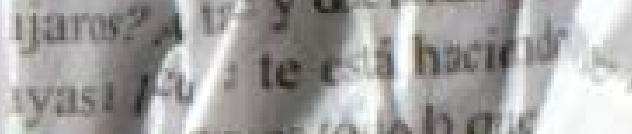
lenty poo es to: bo gis Ahneias del abo.

Titulo: "Son para verte mejor"

Referencia: Grimm, cuentos de los hermanos Grimm. Ed. EMU Año: 2013

Serie: Libros de Poemas

Técnica: Arte objeto; intervención de libro

Medidas: 28 × 21 × $6.5 \mathrm{cms}$.

Foto: Pablo Cuéllar

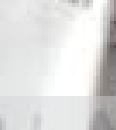

Coperucita Roja

nuyor y más hermosice más ceenestar ys fue alejando cada, is del caincogi vinadas flores.

Pero el lobo no se

a hacasa de la abuelit tivo y sc icanó ó a la pura ¿Quien es? - pre la abuelin Soy Caperucita R

$\rightarrow$ spondió el lobo, imh te trainon No puedo levala a de a in

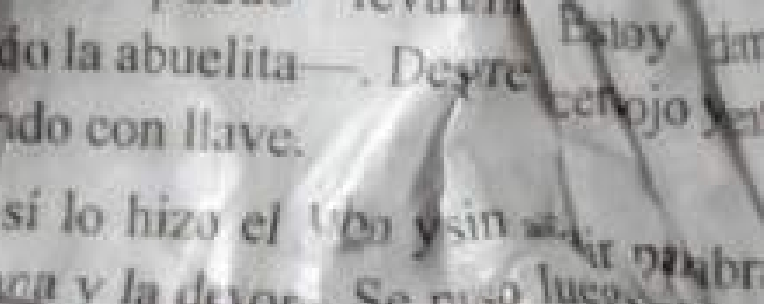
kana y la devor. Se puso luegiver. e colcoó la cofia on la cabja, $s$ cortinas.

$s$ tanto, la niña habia ido rec

fue posible llevar más, rear
Se extrañó mucbo al hallan gYabierta de par en par, pero pene t' que hubietr sucedido nasia. que todo va muy raro y le sa of extrafta sensación. Dijo: dias -pero no obtuvo res (29leq, a la ima, descorrió las ce

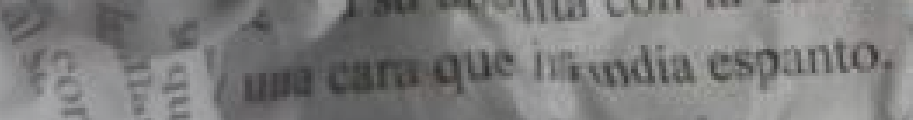
5. है. $\mathrm{f}$, abuelita! Jor que liunes oft ira poder oirte mejor. 


\section{Programa bracero como fenómeno migratorio: análisis de caso en el Estado de Nuevo León}

\section{Bracero program as a migratory phenomenon: case analysis in the State of Nuevo León}

David Saúl Coronado Ortega

\section{Resumen}

La implementación del Programa Bracero sirve como referente en los tratados internacionales dentro del ámbito laboral, previo a la creación de la Organización de las Naciones Unidas (ONU) y a la Declaración de los Derechos Humanos (DH), los cuales son inherentes a las personas y con antecedentes en la declaración de la Revolución Francesa. A través de esta investigación se buscó conocer las vivencias y formas de contratación, de las personas que fueron parte del programa bracero, siendo del Estado de Nuevo León. "A su vez el mismo trabajo nos aporta una remembranza histórica con quienes formaron parte del programa bracero durante 22 años. Ese programa llevó de manera legal durante veintidós años a más de cuatro y medio millones de trabajadores mexicanos" (Alba, 1980, p.358, Morales, 1989, p.201, García, 2007, citado en Smith, 1995, p.123).

Palabras clave: migración, historia, tratados, trabajo, contratación, bracero.

\section{Abstract}

The implementation of the Bracero Program serves as a reference in international treaties within the workplace, prior to the creation of the United Nations Organization (UNO) and the Declaration of Human Rights (DH), which are inherent to people and with antecedents in the declaration of the French Revolution. Through this research, we sought to know the experiences and hiring methods of the people who were part of the bracero program, being from the State of Nuevo Leon. "In turn, the same work gives us a historical remembrance with those who were part of the bracero program for 22 years. "In turn, the same work gives us a historical remembrance with those who were part of the bracero program for 22 years. That program legally took over twenty-two years to more than four and a half million Mexican workers" (Alba, 1980, p.358, Morales, 1989, p.201, García, 2007, quoted in Smith, 1995, p. 123).

Keywords: migration, history, treaties, work, hiring, bracero. 
$\mathrm{E}$ programa bracero que se implementó a mediados del siglo XX, abría oportunidades de poder trabajar la tierra (también fue un programa destinado a trabajadores ferrocarrileros, ver al respecto el artículo de Bárbara Driscoll de 1985), aunque esa no fuera de ellos y se encontraran en un país distinto, pero al final del día se obtenía una remuneración económica que no podían encontrar en su país de origen.

La migración entre México y Estados Unidos es un fenómeno centenario y uno de los flujos migratorios internacionales contemporáneos más representativos. De esta forma y cuando se trata de dinámicas con profundas raíces históricas, de países vecinos y de fronteras móviles, no tiene mucho sentido determinar el momento en que se inició el proceso. (Durand \& Massey, 2003)

Al tiempo que se involucraban en todo el proceso que conlleva dicha migración como acercamiento a costumbres diferentes, idioma, la discriminación de la época y las condiciones precarias de trabajo que no preveía otorgar dicha medida institucional entre los países involucrados. En este programa los jornaleros tenían contratos con duración de 45 días 0 de meses, todos ellos eran nuevamente contratados en la frontera sin necesidad de salir de EEUU para regresar a los ranchos donde los rancheros los esperaban para continuar trabajando en la pizca.

El programa Bracero no puede analizarse como un caso aislado, ni excepcional, sino como parte de un proceso histórico del que existen diversas perspectivas, sobre todo desde la óptica social e histórica entre México y EEU, así como económico cuando se parte del desarrollo del sistema capitalista tanto en el nivel regional como en el contexto total global la expansión internacional de las relaciones de mercado y la persecución global del liberalismo económico (Sandoval, 2015, p.78, Extraído de Tras los braceros entre la teoría y la realidad).

Durante décadas México se consideró un país de emigración, un país donde los estudios, análisis, referencias y ejemplos para hablar de migración se deban siempre en el marco de la experiencia de los mexicanos radicados en Estados Unidos. Es interesante como México ha marcado una pauta con la migración con su vecino del norte EEUU. Desde una visión social y política sin dejar de cada cierto tiempo analizar cómo ha ido cambiando la dinámica migratoria.

Hablar de migración era, por tanto, sinónimo de discusión sobre la situación de los nacionales allende las fronteras, la incorporación de los nuestros a esa sociedad vecina, y los desafíos y las controversias que su sola presencia causaba a una sociedad como la estadounidense, ambigua siempre ante este flujo constante desde hace más de un siglo: por un lado, felices de recibir la mano de obra barata y abundante, por el otro, críticos y hostiles ante su sola presencia. (Calderón, 2014, P. 324, Extraído de Migraciones Internacionales, Crisis y Vulnerabilidades)
El objetivo de esta investigación es analizar y abordar, cómo se dio el proceso de migratorio los braceros de la población de Nuevo León, saber qué tipo de políticas públicas han sido aplicadas para ahora, que son ya una población de la tercera edad, estén protegidos (servicios de salud, pensiones, entre otros). Estudiar el proceso migratorio y la condición de vida de los braceros al llegar a los Estados Unidos. Analizar los principales elementos políticos que afectan a la población braceros y su marginación actual.

Para poder obtener los resultados necesarios y que ofrecieran información que reflejara de la forma más fiel posible las vivencias de los braceros, se usó el método de muestreo conocido como bola de nieve (muestras en cadena o por redes) y entrevistas semi estructuradas con la finalidad de que fuera una forma de acercamiento más directo con los involucrados en el programa bracero y al ser contestadas las preguntas servía para que ellos mismos fueran contando anécdotas, recuerdos, historias de lo que vivieron siendo parte del programa bracero.

El significado de cada vivencia o experiencia resulta central.

En las biografías y las historias de vida, el investigador debe obtener datos completos y profundos sobre cómo ven los individuos los acontecimientos de sus vidas y a sí mismos. En las historias de vida y biografías es esencial tener fuentes múltiples de datos. (Sampieri, Fernández-Collado, Baptista 2006, pp. 619-621, Metodología de la Investigación, Cuarta Edición) 
El programa Bracero no puede analizarse como un caso aislado, ni excepcional, sino como parte de un proceso histórico del que existen diversas perspectivas".
Por medio de la entrevistas semi estructuradas se obtuvieron las vivencias al momento de ingresar el programa bracero, resaltando que son personas de la tercera edad oscilando entre 75 y 80 años. Situación que hacía un poco más complicado que recordaran fechas, momentos y situaciones en tiempo y forma precisos. Lo que arrojó información de suma importancia, recuperando una historia olvidada de Nuevo León de ciudadanos que formaron parte del programa bracero a nivel nacional, teniendo un registro aproximado de

do llega con el boom industrial y la construcción de casas en Nuevo León.

El Señor Juan Rodríguez:

Argumenta que a pesar de haber logrado tener un trato directo con el patrón, él debía comer en la cocina y que no podía asistir a los bailes organizados por los dueños de otros ranchos, aun y que fuera acompañando a su patrón y que al llegar a una gasolinera y un mexicoamericano los iba a atender, decía no atender a mexicanos.

- "Era lo más visible durante el tiempo que fui bracero".

5 mil personas (Información obtenida por Alianza bracero proa) de Nuevo León $y / 0$ que fueron contratados desde aquí. En este artículo se enfoca en dos de las entrevistas que se aplicaron y que ofrecen información interesante referente a las vivencias de ingresar al programa bracero:

El Señor Eulalio:

Él decidió ingresar al programa bracero para ser útil y aprender cosas, tenía 20 años cuando se fue y, que el irse, le ayudó en su maduración y saber hacer cosas para lo que se le necesitara (plomería, electricidad, trabajos diversos) aparte la pizca que nunca dejo de hacer, pero que, al regresar con lo aprendido allá, pudo dedicarse a esos oficios con un negocio propio. Y que con lo aprendido decide quedarse en Monterrey para ser contratista de servicios y no tener que trabajarle a nadie, se viene con sus hijos de Venados, San Luis Potosí, tenía entre 22 y 24 años; él ya venía inscrito de la lista de Gobernación, para ser contratado en la Ciudad de Monterrey. Tuvo 3 contratos de 45 días cada uno, en un tiempo aproximadamente de medio año siendo bracero y, es a finales de los 50, cuan-
También platica que tuvo la oportunidad de trabajar en una empresa en tiempo posterior al programa bracero como a 70 millas de Houston.

Nos platica que él tuvo 6 contratos con duración de 3 meses cada uno. Era fácil obtener la mica y poder ingresar al programa bracero, con tener más de 19 años, cartilla liberada y tu acta de nacimiento era como se podía obtener la mica e ingresar al programa bracero.

- "Siendo del sur del estado de Nuevo León (Arramberi) vi como por Ciudad Juárez pasaron más de 5 mil personas contratadas para el programa bracero".

Así mismo, nos platicaba que él tuvo la oportunidad de aprender el manejo de tractores, camiones de $3 / 5$ toneladas para el uso correcto de lo recolectado en la pizca, pero que el aprender el uso del tractor no lo exentaba de estar en la pizca de tomate, chile o pepino. Todas estas contrataciones fueron efectuadas dentro del campo militar 7-B que se encuentra a un costado de Ciudad Universitaria (UANL). 
Para un desarrollo eficiente del marco teórico dentro de esta investigación, fue necesario analizar diferentes teorías para tener un espectro más amplio con relación a que se deseaba conocer del proceso migratorio y cómo podía ser relacionado con lo vivido durante el programa mismo y cómo había sido abordado por diferentes autores en el desarrollo de sus teorías migratorias.

También es interesante reaprender que fue necesario conocer el tiempo y espacio ocupado por el programa bracero y cómo esto género que hoy en día sea interpretado como algo anecdótico y quizá sin un valor real de un acontecimiento histórico, pero que es un problema migratorio que cuenta con mucho presente y futuro y que desafortunadamente no parece tener un final feliz para los que fueron parte del programa bracero.

La experiencia migratoria se socializa, se aprende desde la más pequeña infancia. En particular entre muchos adolescentes y hombres jóvenes, la salida hacia el norte es alentada por la valoración social y la expectativa de acumular prestigio y poder entre familiares, en la comunidad y en grupos de pares. Al igual que las trayectorias, los actores de la migración son múltiples: ex braceros, retornados, deportados, polleros o coyotes, indocumentados, profesionistas y empresarios cosmopolitas. Como lo señalábamos, el cambio más notable en los patrones migratorios es la diversificación de los flujos (París Pombo, 2012, p.19).

Piñeiro y Zapata (2013) establecen que la migración de mexicanos hacia
Estados Unidos no es un fenómeno nuevo, tiene más de 100 años de antigüedad. La magnitud y sus efectos son temas polémicos, no solo a nivel gubernamental sino también en el académico y el social, tanto en México como EE.UU. Del periodo abarcado del año 1900 hasta el año 1950, el desplazamiento de migrantes laborales hacia EE.UU. apenas rebaso el millón de personas. Inicialmente la dinámica seguía un flujo circular, es decir, que la mayoría de los mexicanos que iban a trabajar en los campos agrícolas de Estados Unidos se asentaba por un periodo de entre siete y nueve meses y regresaba a su lugar de origen para estar en compañía de sus familiares durante las celebraciones de fin de año. Después en enero o febrero, regresaban a EE.UU. a trabajar.

El fenómeno migratorio es también visto como un medio para asegurar la subsistencia, donde en los lugares de origen aspectos tales como la pobreza y la falta de oportunidades constituyen los principales factores que la motivan, así como las grandes diferencias salariales entre los países expulsores y los receptores.

(Acharya y Salas, p.222).

La migración es uno de los fenómenos en la sociedad mexicana de mayor significancia siendo México el sexto Estado Nación que más población ha expulsado al día de hoy en términos absolutos (BM, 2014). Sin embargo, la frontera norte de México tiene una peculiaridad sobre el resto de las otras fronteras del mundo, por ser la más transitada, y por ende, la de mayor dinámica migratoriacon EEUU" (OIM, 2014).

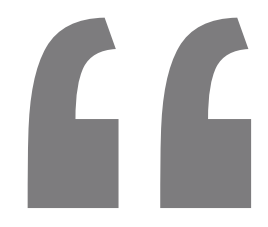

Piñeiro y Zapata (2013) establecen que la migración de mexicanos hacia Estados Unidos no es un fenómeno nuevo, tiene más de 100 años de antigüedad". 
Este trabajo desmitifico la idea generalizada hasta nuestros días de que el programa Bracero fue una alternativa que beneficio a los trabajadores mexicanos y de que, por lo tanto, fue una opción de política exterior muy positiva para México. Esto se tradujo en prácticas de explotación y abusos de poder por parte de estos y del propio gobierno de Estados Unidos. Este trabajo da una idea clara de la complejidad del fenómeno migratorio del raterismo y la transición del México rural al México urbano (Bustamante, 2014).

Al mismo tiempo, ayuda saber qué tan beneficiario fue el programa bracero y cómo fue el desarrollo de ciudades fronterizas a diferencias de ciudades del área metropolitana en Nuevo León. Aunque lo relevante de toda esta investigación era conocer el trato que recibieron los braceros durante la estancia 0 sus contratos en territorio estadounidense, existe la hipótesis de que al hacer las entrevistas fue posible comparar con otras ciudades y cómo vivieron el proceso los braceros estando ya allá.

Conocer cómo fue el desarrollo de ciertas ciudades en comparación con unas ciudades fronterizas, muestra si es tan visible su crecimiento en similitud a ciudades que no son fronterizas, lo evidente en crecimiento, cambio de dinámicas, creaciones de empleos y en cómo la dinámica migratoria es constantemente cambiante y hasta en ciertos puntos hereditaria a las siguientes generaciones.

Existe un marcado error en todos aquellos que se dedican a tratar este tema, en querer hacer radicar las cau- sas del problema en razones de carácter nacional únicamente, olvidando que en él intervienen también los intereses tanto de México (combatir el desempleo, mano de obra barata) como de Estados Unidos (abandono de los campos agricultores, ingreso a la Segunda Guerra Mundial, falta de manos para el trabajo en el campo), aunque para este país no represente algo más que un problema histórico que no involucra al actual gobierno.

Al final se puede concluir que este trabajo debe de servir para no olvidar una problemática social, política, económica y familiar que tiene mucho presente, un futuro muy gris y un presente en lucha. Entonces no se puede hablar de la problemática como algo que ya pasó, que no ha podido ser resuelto y que será olvidado con el paso de los años y que existen ciudades con una comunidad de braceros muy amplia y que desean ser escuchados, atendidos, pero ante todo que haya justicia social de parte del Estado con ellos.

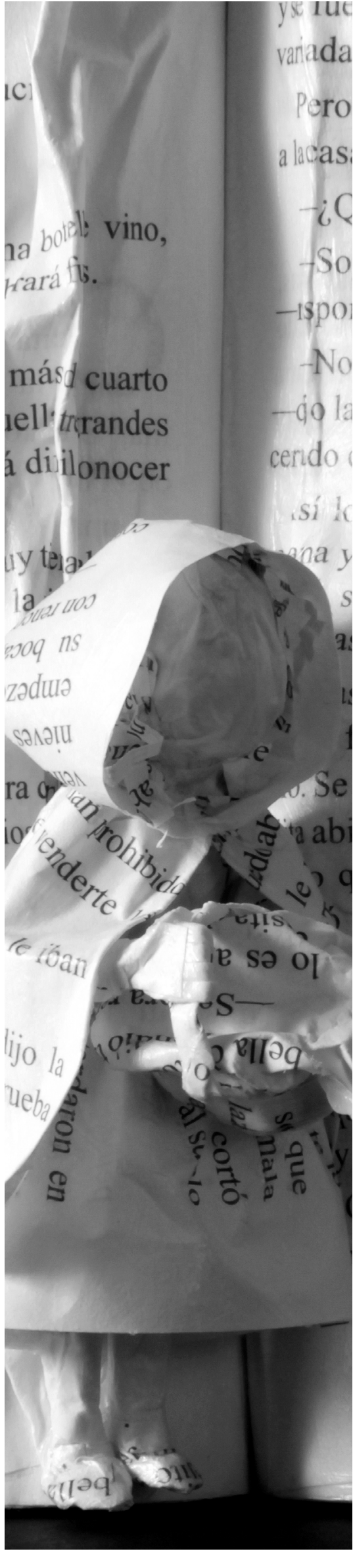

Presencia Universitaria 


\section{Referencias}

Acharya, Arun Kumar (2013). Prefacio. Brittite Lamy (COORD.) Impactos Socioculturales de la Migración. México: Miguel Ángel Porrúa.

Durand Jorge \& Massey Douglas S (2003). Clandestinos. Migración México- Estados Unidos en los Albores del Siglo XXI: Universidad de Zacatecas. Primera edición 2003.

Gómez Walteros, Jaime Alberto (2010). La Migración Internacional: Teorías y Enfoques, Una Mirada Actual Semestre Económico [en línea] 2010, 13 (Enero-Junio).

Grijalva, Aidé \& Arriaga, Rafael (2015). Tras los Pasos de los Braceros "entre la teoría y la realidad". Instituto de investigaciones sociales, Mexicali, Baja California.

Anguiano Téllez, María Eugenia \& Cruz Piñeiro Rodolfo/Coordinadores. (2014). Migraciones internacionales, crisis y vulnerabilidades: Perspectivas comparadas/Tijuana: El Colegio de la Frontera Norte, 2014.

Organización Internacional para las Migraciones (OIM) (2014). Hechos y cifras (2014) Ver http://oim.org.mx/hechos-y-cifras-2.

Ojeda Gómez Mario (2014). La produccion de los trabajadores emigrantes. Tijuana: El Colegio de la Frontera Norte; México, D.F.: Universidad Nacional Autónoma de México, 2014.

París Pombo María Dolores (2012). Migrantes, desplazados, braceros y deportados: experiencias migratorias y prácticas políticas/Tijuana: El colegio de la Frontera Norte; Ciudad Juárez, Chihuahua: Universidad Autónoma de Ciudad Juárez; México, D.F.:UAM-Xochimilco, Primera Edición 2012.

Smith, Emma (2011). Año 12, núm. 26, revista electrónica; Centro de enseñanza para extranjeros, UNAM.

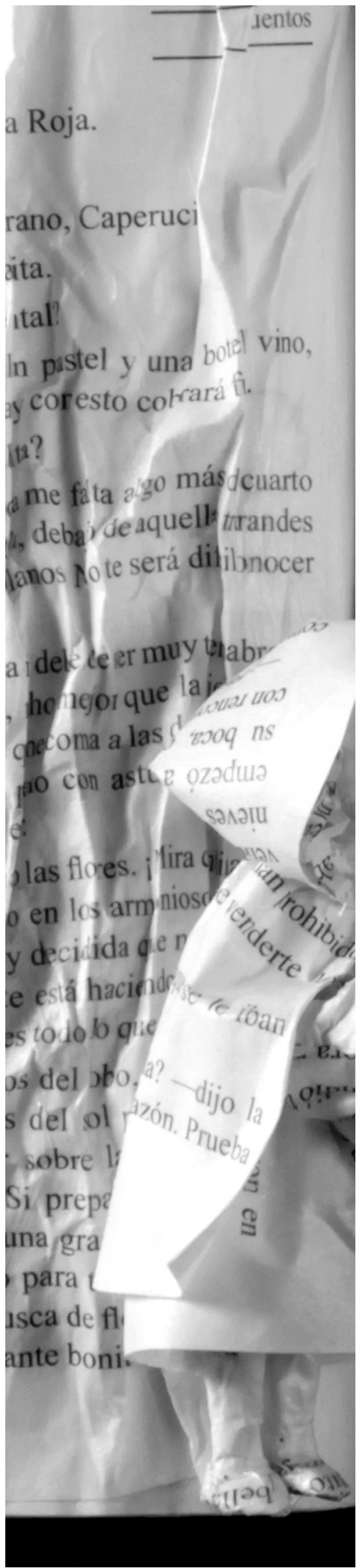




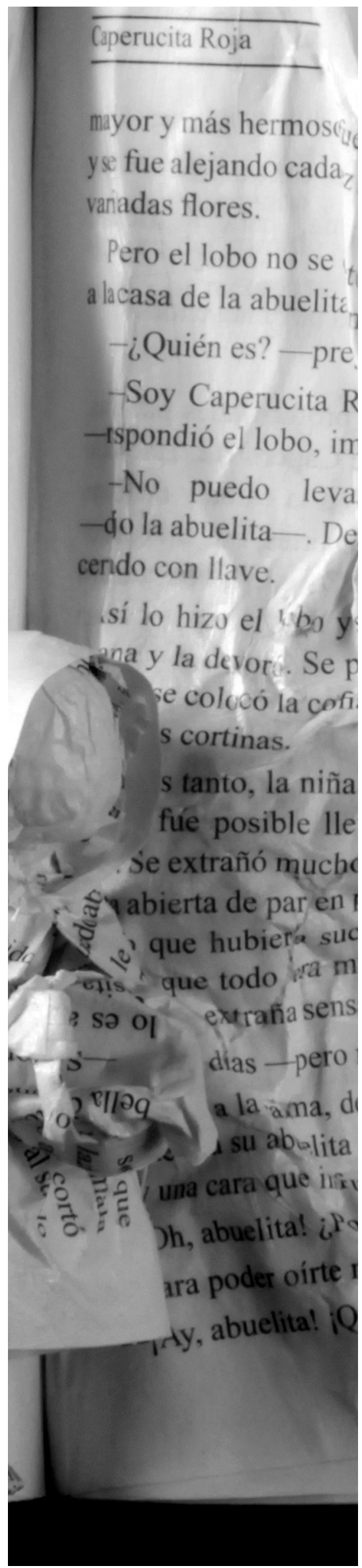

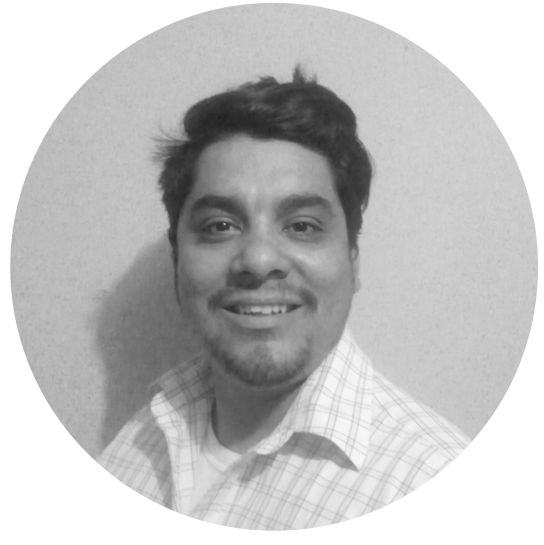

\section{David Saúl \\ Coronado Ortega}

Es Licenciado en Gestión y Desarrollo Municipal por la Facultad de Ciencias Políticas y Relaciones Internacionales de la Universidad Autónoma de Nuevo León. Tiene Maestría en Ciencias Sociales por el Instituto de Investigaciones Sociales de la UANL. Docente Titular en Universidad Tec Milenio Campus Lázaro Cárdenas nivel bachillerato. Asesor en la Alianza de Usuarios A.C.

Correo electrónico:

davidsaul_c@hotmail.com
Recibido: 30/08/2017

Aceptado: 20/11/2017 